\title{
Design and Testing of a Textile EMG Sensor for Prosthetic Control
}

\author{
Luisa M. Arruda ${ }^{10000-0002-5172-459 x]}$, Alexandre Calado ${ }^{\text {[0000-0002-2725-1067] }}$, Rachel S. \\ Boldt ${ }^{[0000-0002-6309-1419]}$, Yao Yu ${ }^{1}$, Helder Carvalho ${ }^{{ }^{[0000-0003-0472-1107] ~}}$, Miguel A. F. \\ Carvalho $^{{ }^{[0000-0001-8010-6478] ~}}$, Fernando B. N. Ferreira ${ }^{{ }^{[0000-0003-1270-8041] ~}}$, Filomena Soa- \\ res $^{2^{[0000-0002-4438-6713]}}$ and Demétrio Matos ${ }^{3^{[0000-0003-4417-6115]}}$ \\ ${ }^{1}$ Centro de Ciência e Tecnologia Têxtil, University of Minho, Guimarães, Portugal \\ ${ }^{2}$ Centro Algoritmi, University of Minho, Guimarães, Portugal \\ ${ }^{3}$ Instituto Politécnico do Cávado e do Ave, Barcelos, Portugal \\ luisamendesarruda@gmail.com
}

\begin{abstract}
Nowadays, Electromyography (EMG) signals generated by the amputee's residual limbs are widely used for the control of myoelectric prostheses, usually with the aid of pattern-recognition algorithms. Since myoelectric prostheses are wearable medical devices, the sensors that integrate them should be appropriate for the users'daily life, meeting the requirements of lightness, flexibility, greater motion identification, and skin adaptability. Therefore, this study aims to design and test an EMG sensor for prosthetic control, focusing on aspects such as adjustability, lightness, precise and constant signal acquisition; and replacing the conventional components of an EMG sensor with textile materials. The proposed sensor was made with Shieldex Technik-tex P130+ B conductive knitted fabric, with $99 \%$ pure silver plating. EMG data acquisition was performed twice on three volunteers: one with the textile sensor, and other with a commercial sensor used in prosthetic applications. Overall, the textile and the commercial sensor presented total average Signal-to-Noise Ratio (SNR) values of $10.24 \pm 5.45 \mathrm{~dB}$ and $11.74 \pm 8.64 \mathrm{~dB}$, respectively. The authors consider that the obtained results are promising and leave room for further improvements in future work, such as designing strategies to deal with known sources of noise contamination and to increase the adhesion to the skin. In sum, the results presented in this paper indicate that, with the appropriate improvements, the proposed textile sensor may have the potential of being used for myoelectric prosthetic control, which can be a more ergonomic and accessible alternative to the sensors that are currently used for controlling these devices.
\end{abstract}

Keywords: Textile Electrode, EMG, Prostheses.

\section{Introduction and State-of-the-art}

The scientific community has set goals for advancements in the field of upper limb prosthetic devices, in which specific requirements must be reached: simultaneous, independent, and proportional control of multiple degrees-of-freedom with acceptable 
performance and adequate response times [1]. To this end, the importance of proper acquisition and processing of electromyographic (EMG) signals is required. For the success of future prostheses, the sensors that integrate them should be qualified for the daily life of users, meeting the requirements of lightness, flexibility, greater motion identification, long battery life and easy skin adaptability. This study starts from the motivation to develop an ergonomic, viable, flexible sensor that contributes to a more effective and comfortable control of myoelectric prostheses.

In this sense, the state of the art points out that one of the most used ways to control prosthetic devices is through the residual limb muscles' EMG signals. In this technique, conductive electrodes detect small electric potentials generated by the exchange of ions through the muscular membranes. The obtained signal is known as myoelectric, and the conversion methodology is called electromyography. Observing these signals and their properties (frequency, amplitude, power and rise time) allows both identifying abnormalities in muscle contraction as well as identifying and classifying different movements performed by the body. More specifically, it is possible to use pattern recognition algorithms to classify the signals obtained by EMG and allow prosthetic devices to recognize such patterns and respond in an expected way [2].

An example of this application is the Myo Armband [3]. Launched commercially in 2013, it is a wearable system for multimedia applications such as games and screen display control that fuses eight channels of EMG sensor information with inertial sensors to achieve gesture recognition. Being a bracelet that is adjustable for various arm dimensions, and that can be positioned in several points to obtain simultaneous signals from distinct forearm muscles, it is suggested as an affordable alternative for myoelectric prosthesis control $[3,4]$.

With the advent of wearable systems, the interest in using smart textiles in the medical field is intensifying [5,6]. Textiles are more flexible and adapt better to different designs when compared to conventional electronic structures. Specifically, for prosthesis control, research has been done on textile sensors whose electrodes are composed of conductive inks [7], nickel-plated conductive fabric [8] or even through knitting electrodes made of silver wire [9]. All these studies compare the signals obtained by textile sensors with conventional $\mathrm{Ag} / \mathrm{AgCl}$ gel electrodes. For instance, in one study knitted textile electrodes were used, and the signal to noise-ratio obtained was on average $17.97 \mathrm{~dB}$, better than the $13.3 \mathrm{~dB}$ obtained with disposable electrodes [9].Textile electrodes present several advantages: flexibility, wearability, breathability and easy integration into garments such as sockets used on residual limbs.

The work herein described aims to assess the reliability of one type of construction for textile electrodes, when compared to an EMG sensor already established in the prosthetic market. Furthermore, a comparison of the activation of individual muscles by specific gestures is made, in order to assess the possibility of gesture recognition. 


\section{Materials and Methods}

\subsection{Sensor design}

The sensor was designed to fulfill the characteristics of a wearable piece, therefore, the following requirements were listed: adjustability, in order to be adaptable to various body types; lightness, considering that one of the factors of prostheses rejection is the weight of the medical devices $[10,11]$; precise and constant signal acquisition, in order to be comparable to sensors already used by the prosthetic market; and replacing the conventional components of a sensor with a textile material, thus bringing it closer to a garment.

The guidelines of the Surface Electromyography (sEMG) for the Non-Invasive Assessment of Muscle Project (SENIAM) were followed [12]. These guidelines are based on recommendations made by countries of the European Union included in the program of Health and Biomedical Research (BIOMED II). The recommendations focus on aspects such as: sensor design (shape, size, distance between electrodes, raw material to use and how to build it); placement of sensors (skin preparation, position and fixation); and location of the muscles for sensor positioning.

To meet the adjustability requirement, the sensor was made of an elastic textile structure, $65 \%$ polyester and $35 \%$ elastane, with $30 \mathrm{~cm}$ of length. A touch fastener with $12 \mathrm{~cm}$ was used, which allowed the same sensor to be tested on volunteers whose forearm width ranged from 21 to $30 \mathrm{~cm}$.

Regarding electrode dimensions, the first prototype was composed of circular shaped electrodes, $10 \mathrm{~mm}$ in diameter and $20 \mathrm{~mm}$ of inter-electrode distance.The material used for the construction of the electrodes was the Shieldex Technik-tex P130 $+B$ conductive knitted fabric $(78 \%$ Polyamide $+22 \%$ Elastane), with $99 \%$ pure silver plating. The conductive fabric was applied to the aforementioned band with a thermoplastic adhesive (Bemis 5254) at $170^{\circ}$ and 5.5 bar for 20 seconds on the heat press. However, when performing the contact tests whose protocol is described below, only noise was obtained.Therefore, the size of the electrodes was gradually increased, and the shape was changed. The best results were obtained with $2 \times 5 \mathrm{~cm}$ rectangular electrodes, using two electrodes and a reference electrode positioned at the most distal part of the forearm (Fig.1). Thermoplastic polyurethane (TPU) tape and film were used around the electrodes to isolate the detection area for better sensor performance, as previous work has shown [13] (Fig.2). The textile sensor presented a weight of $17.48 \mathrm{~g}$. 


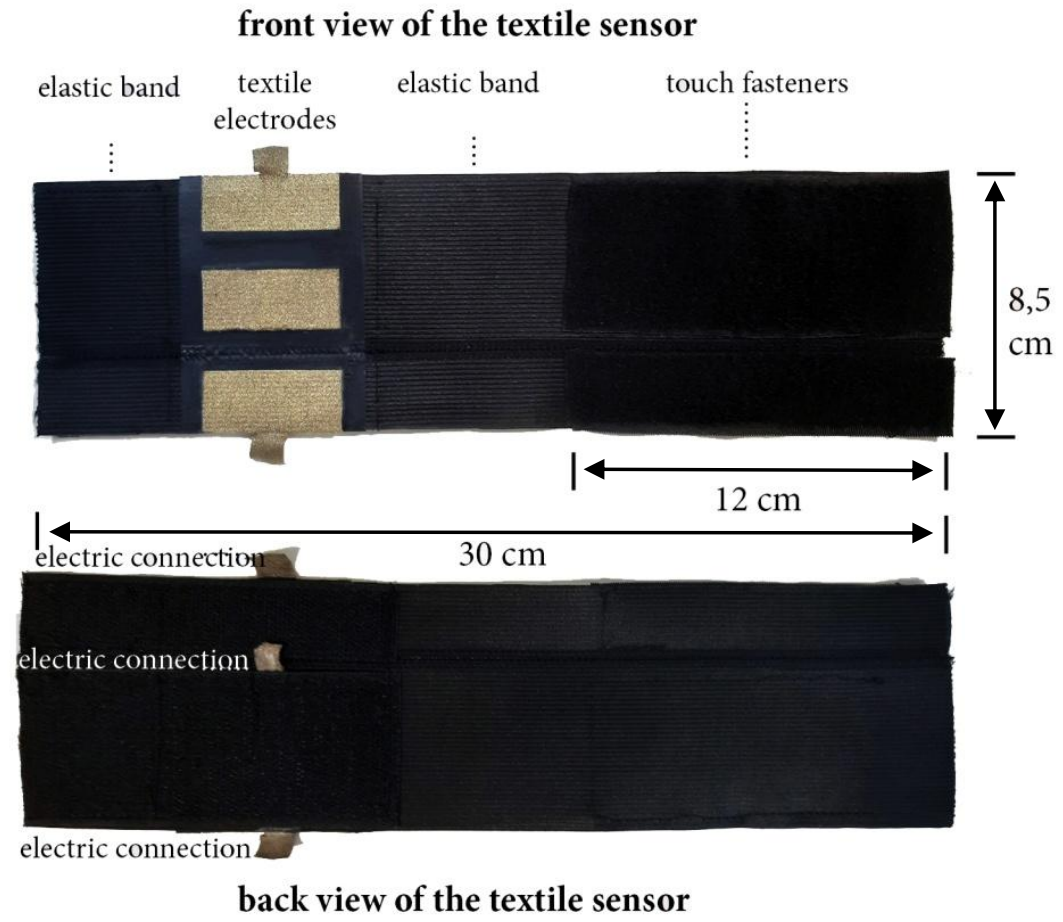

Fig.1. The figure shows the design and dimensions of the elastic band with the integrated textile electrodes.

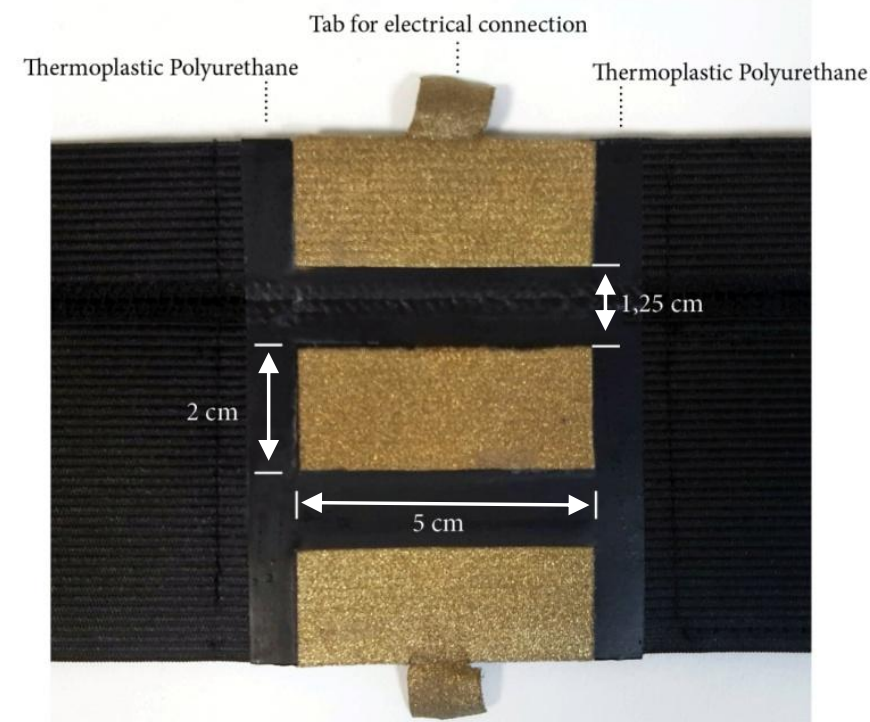

Fig.2. The figure shows the expanded view of the textile sensor. 
In order to have a means of comparison, besides acquiring EMG data with the proposed textile sensor, a commercial sensor was also used. This way, it was possible to grasp a clearer idea of how good the textile sensor performs. For the purposes of this work, an Ottobock 13E68 sensor was used. This sEMG sensor is circular and measures $27.5 \mathrm{~mm}$ wide by $13.5 \mathrm{~mm}$ high, weighing $9 \mathrm{~g}$, according to the manufacturer (Fig.3). It is a bipolar model with amplification, internal filtering and enveloping. It is also a sensor used for myoelectric control of prostheses.

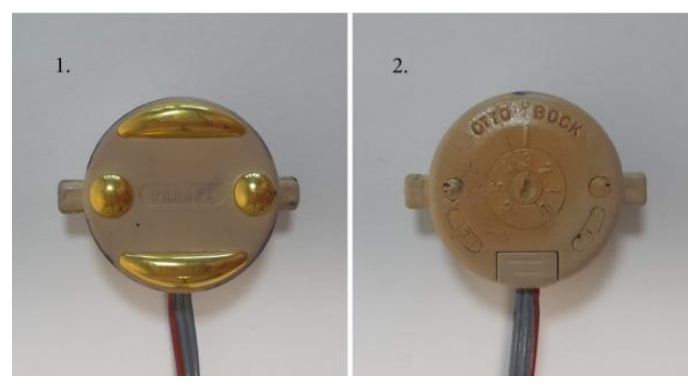

Fig.3. The figure shows the conventional sEMG model 13E68, manufactured by Ottobock, where 1 is the front view and 2 the back view.

\subsection{Subject Information}

Three subjects (1 male and 2 female), with 25, 30 and 33 years old, respectively, without any neurological and muscular pathology, were selected to perform the tests. All individuals provided written informed consent and gave permission for the publication of photographs for scientific and educational purposes. The male volunteer will be referred here as V1 and had a $26.5 \mathrm{~cm}$ width forearm, volunteer V2 had a $21 \mathrm{~cm}$ width forearm, and volunteer V3 had $25 \mathrm{~cm}$ width forearm.

\subsection{Protocol and EMG Data Acquisition}

The EMG data acquisition experiment was performed with each subject. This experiment was composed by two parts. In the first part of the experiment, EMG data was acquired through textile sensors positioned alternately in the Flexor Carpi Radialis, Flexor Carpi Ulnaris, Extensor Carpi Ulnaris and Extensor Carpi Radialis muscles. These muscles were selected due to their active contribution to finger and hand movement, as well as their location on the forearm, as they are superficial muscles, which means that the generated signals are easier to detect with surface electromyography (sEMG). In order to identify the location of such muscles in a tactile manner, standard instructions were followed according to the literature [14]. Thereafter, in the second part of the experiment, the Ottobock sensor was positioned at the same locations as the textile sensor (Fig.4). There was a rest between each test. The time interval between the two parts of the experiment was of one to two days, depending on the availability of volunteers. 


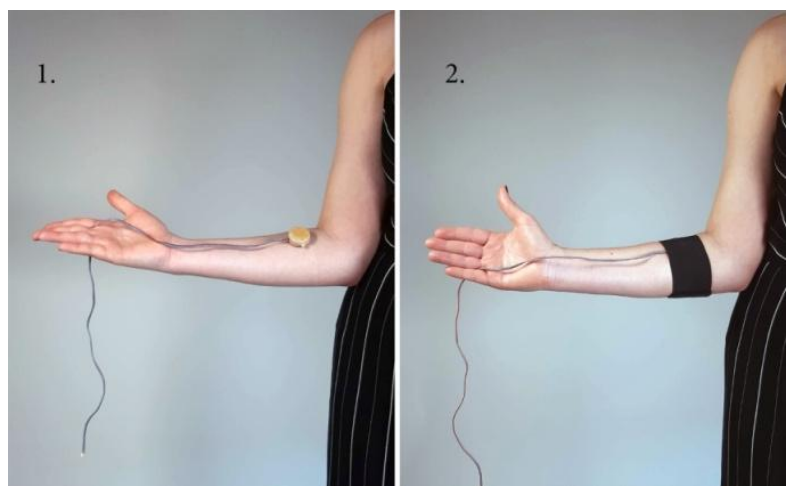

Fig.4. This image depicts how the commercial sensor (Ottobock) was placed on the volunteers'right forearm with an elastic band similar to the one used with the textile sensor.

Six gestures were listed for the tests, namely: Fist (G1), Spread (G2), Wave-in (G3), Wave-out (G4), Pinch (G5), Shoot (G6), (Fig.5). Each gesture, in turn, was repeated five times, with an interval of $5 \mathrm{~s}$ between each action. It was recommended that the volunteers apply their highest level of isometric force production.

The data acquisition system consisted of a NI USB-6229 data acquisition board with software in LabVIEW. Whilst the signals from the Ottobock sensor were acquired directly, the textile armband was used with the Myoware AT-04-001 muscle sensor for signal conditioning, being its output connected to the data acquisition board. All signals were acquired using a sampling frequency of $1 \mathrm{KHz}$.

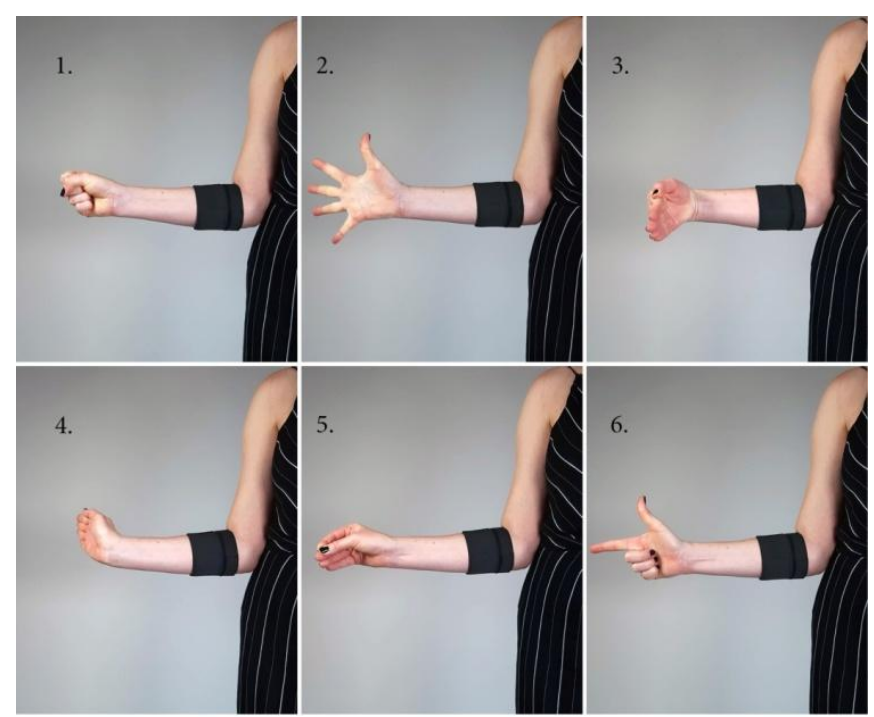

Fig.5.The image shows the six gestures performed by the volunteers: Fist (G1); Spread (G2); Wave-in (G3); Wave-out (G4); Pinch (G5); Shoot (G6). 


\subsection{Signal processing}

The sensors manufactured by Ottobock, such as the 13E68, are optimized for prosthetic/myoelectric control purposes, featuring on-board analog amplification, filtering and enveloping, whereas the proposed textile sensor's signal is "raw", i.e. it lacks any signal processing.

All signal processing for the textile sensors was performed digitally in an application developed with LabVIEW. Signals were filtered with a Notch filter at $50 \mathrm{~Hz}$ in order to eliminate the power line noise, and a band-pass filter with cut-off frequencies of 10-450 Hz. The latter were chosen considering that the recommended band-pass frequency range is $10-500 \mathrm{~Hz}[15,16]$. According to the manufacturer's general specifications, Ottobock sensors use a 90 to $450 \mathrm{~Hz}$ passband. For the specific sensor used, a notch filter at 50 or $60 \mathrm{~Hz}$ is specified [14]. However, it was not possible to confirm the exact specification for the sensor.

The final operation to obtain a smoother and more stable signal is enveloping, which was achieved using a moving average filter. A time window of $300 \mathrm{~ms}$ was used, which is appropriate for slow or static activities [16], such as the ones performed during signal acquisition. As previously stated, no signal processing was performed on the EMG signals recorded using the Ottobock sensor.

The quality of the EMG signals was evaluated in terms of the Signal-to-Noise Ratio (SNR), which is a standard method used for this purpose [15]. The SNR can be calculated with Equation 1, where $A_{S}$ is the average signal amplitude and $A_{N}$ is the average noise amplitude.

$$
S N R=20 \log \left(\frac{A_{S}}{A_{N}}\right)
$$

The background noise was considered to the baseline noise and the EMG burst observed while the muscle is contracted is the signal that must be detected.

The SNR was calculated for each recording, which comprised five repetitions of the same gesture (for each sensor position), separated by periods of rest, as described in Section 2.3.

To ease the calculation of the SNR, a semi-automatic algorithm for detection of the activation (onset) and idle periods of muscle activity was implemented, based on the double-threshold detection methods suggested in [15]. In this method, the detection of activation depends on the application of an amplitude threshold, as well as a minimum activation time, in order to reject noise spikes and motion artifacts.

Since each recording starts with approximately 5 seconds of rest, i.e. baseline noise, the baseline was initially defined as the mean voltage of 2 seconds taken of the initial rest period. The user may then adjust this baseline by dragging the cursor drawn by the software to indicate the baseline.

To compute the amplitude detection threshold, a first attempt was made using the suggested criterion [15] in that the EMG signal must surpass a value that represents the $95 \%$ confidence interval $(\mu \pm 1.96 \sigma)$ for baseline activity. However, this method 
seemed to define the threshold too low, and even extending to $(\mu \pm 2.58 \sigma)$ the method revealed not very efficient. It was then decided to calculate the amplitude of the signals and define the threshold as $5 \%$ of the amplitude.

The second criterion was to consider a minimum activation time to consider the activation valid. A default value of 2 seconds was defined.

The automatic detection of the intervals was then graphically represented by cursors. At this moment, it was possible to accept the automatically generated activation/idle intervals, re-generate them with another time threshold, adjust the amplitude threshold manually or adjust the intervals directly, by dragging the cursors.

After this process, SNR was computed based on the generated onsets.

\section{Results}

Fig. 6 to 9 show samples of EMG signals acquired by the Ottobock and textile sensors from the Extensor Carpi Radialis muscle in gesture 4, the one that, generally speaking, produced the strongest signals. In this case, the signals belong to subject V1.

The raw signal obtained with the textile sensor is depicted in Fig. 6, Fig. 7 shows the filtered signal, Fig. 8 the envelope computed with the moving average filter. It is also possible to observe the automatically generated baseline, amplitude threshold values, as well as the activation intervals found. The same can be observed in Fig. 9 for the signal of the Ottobock sensor.

It was possible in about $80 \%$ of the cases to use the automatically generated values directly, whilst in the remaining cases it was necessary to adjust the parameters and recalculate, or to adjust the intervals explicitly.

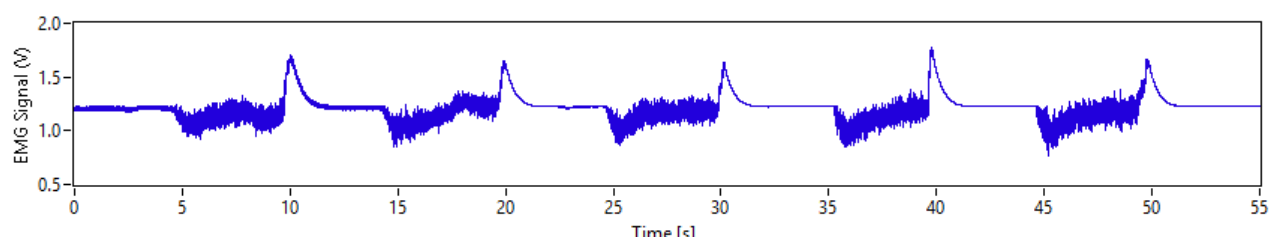

Fig. 6. Raw EMG signal acquired with the textile sensor from subject V1'sExtensor Carpi Radialis muscle in gesture 4

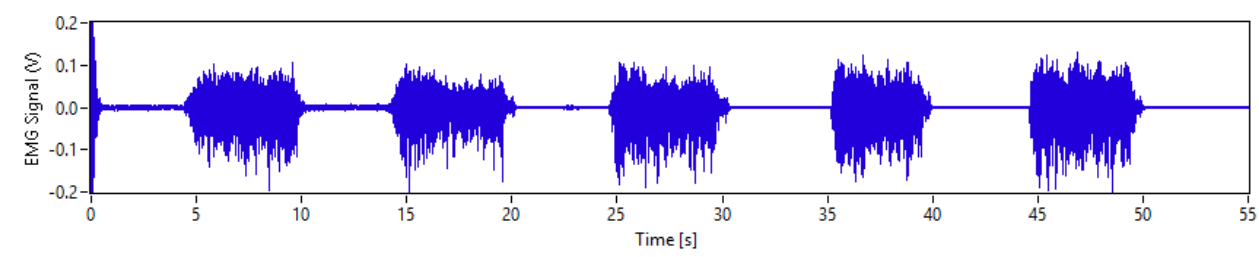

Fig. 7. Bandpass and Notch filtered EMG signal acquired with the textile sensor from subject V1's Extensor Carpi Radialis muscle in gesture 4 


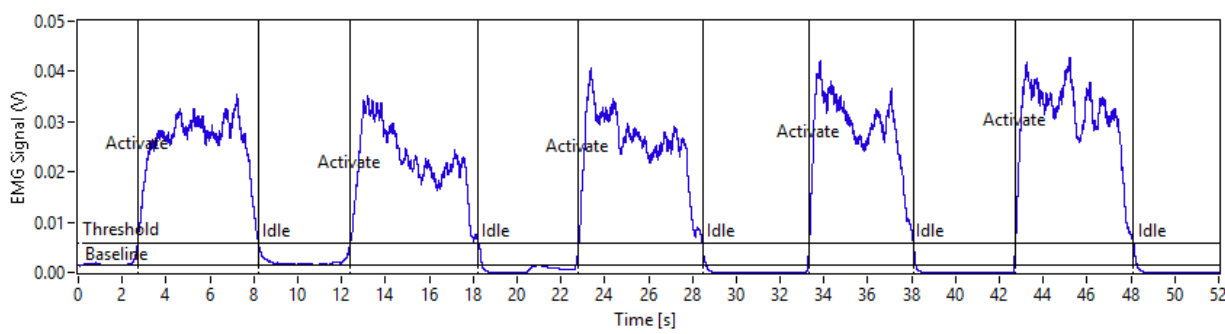

Fig. 8.Enveloped EMG signal acquired with the textile sensor from subject V1's Extensor Carpi Radialis muscle in gesture 4 . It is also possible observe the automatically generated baseline, amplitude threshold values, and computed activation intervals

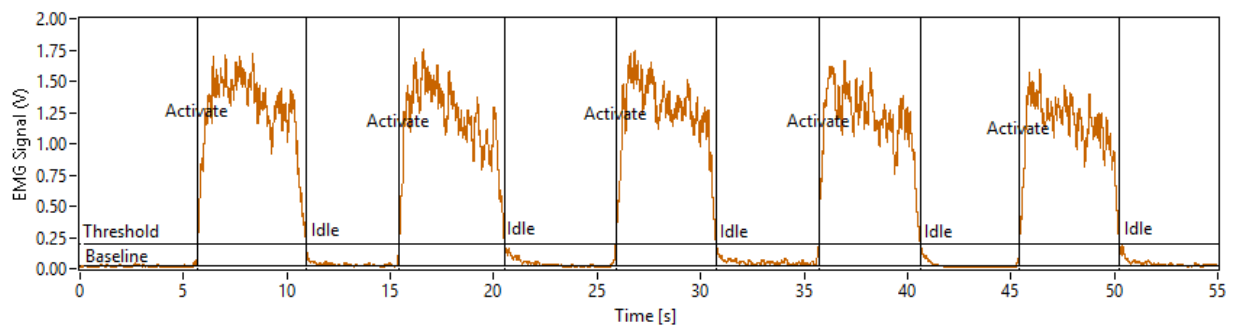

Fig. 9.EMG signal acquired with the Ottobock sensor from subject V1's Extensor Carpi Radialis muscle in gesture 4. As in Fig. 8, it is possible observe the automatically generated baseline, amplitude threshold values, and computed activation intervals

Regarding signal acquisition performed with the textile sensor, some issues were observed during two of the sensor positionings, namely for subject V2, in the position above the Extensor Carpi Radialis, and for the subject V3, in the position above the Extensor Carpi Ulnaris. In both cases, it was not possible to obtain the EMG signal after placing the sensor on the subject's skin surface. This might have occurred due to poor contact between the textile electrode and the skin, or due to some problem regarding the connection between the textile sensor and the EMG MyowareAT-04-001. Regardless, this situation was not verified during the recording performed with the Ottobock sensor. Considering this, the recordings regarding the aforementioned muscles were not considered for the analysis presented in this section.

The results concerning the computation of the SNR are presented in the graphs of Fig.10 and 11, for the textile and the Ottobock sensor, respectively. The graphs represent the average SNR, in $\mathrm{dBs}$, per sensor position (i.e. per muscle), for each of the three subjects. As previously mentioned in this section, the results regarding the textile sensor positioning on the Extensor Carpi Radialis from subject V2 and on the Extensor Carpi Ulnaris from subject V3 were not taken into account. 


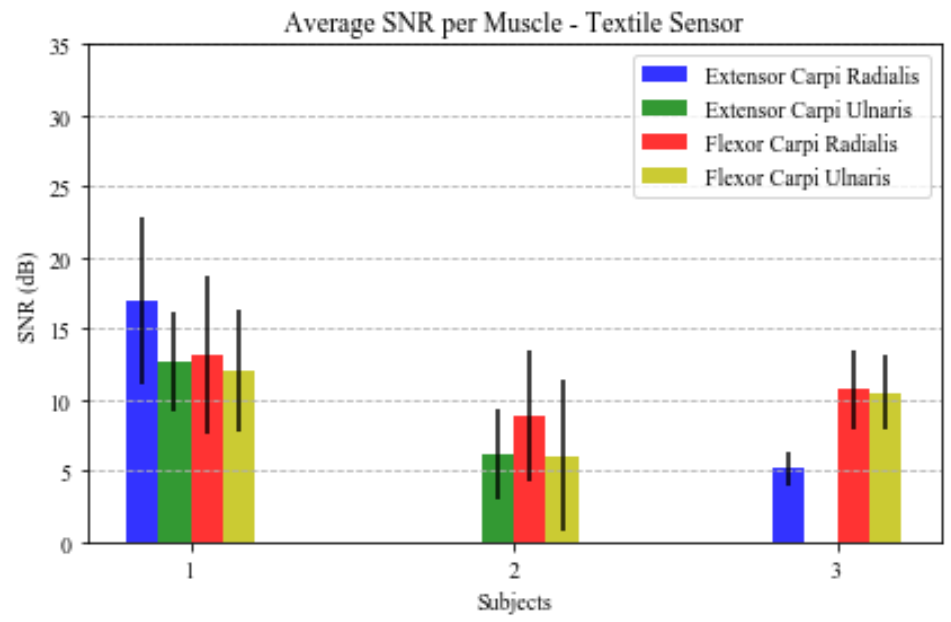

Fig. 10. Average SNR per position for the textile sensor, for each subject

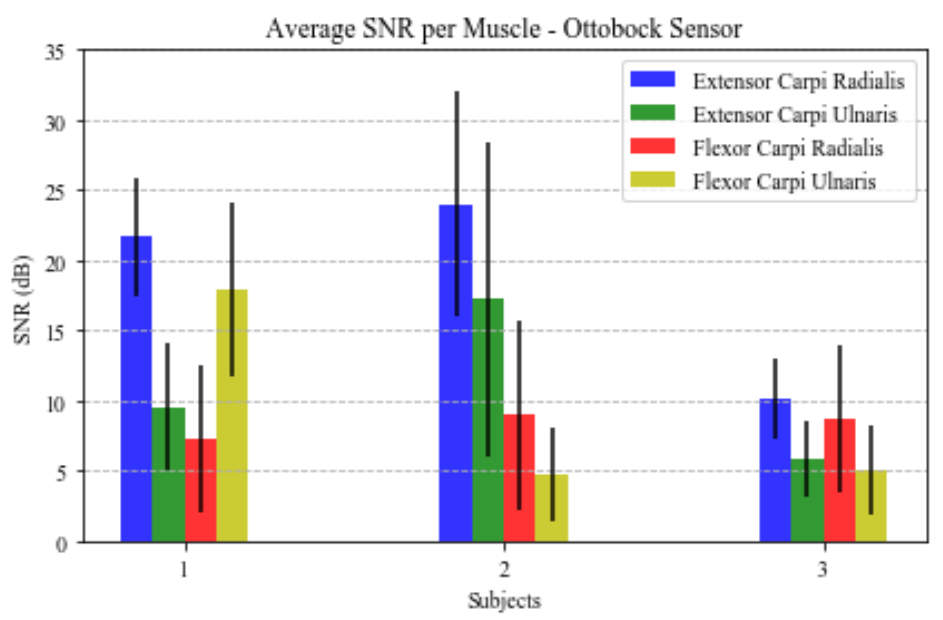

Fig. 11.Average SNR per position for the Ottobock sensor, for each subject

Overall, the total average SNR calculated using the recordings made with the textile sensor was of approximately $10.24 \pm 5.45 \mathrm{~dB}$. As for the Ottobock sensor, the total average was of $11.74 \pm 8.64 \mathrm{~dB}$.

Taking into account the goal of the present work, it is also relevant to validate, even if only empirically, if the EMG signals acquired with the textile sensor have potential to be used for classification with Machine Learning algorithms, as it is traditionally done in typical myoelectric control systems, such as the ones used in myoelectric transradial prostheses. For this, it is important to check if there are distinct EMG patterns for each of the six gestures. Bearing this in mind, the graph depicted in Fig. 12 represents one muscle activation of the first execution of each gesture, pre- 
ceded and followed by 2 second of idle time. The plots correspondent to each muscle were artificially synchronized and overlapped in order to simulate the EMG pattern that could be potentially obtained if four textile sensors were being used at the same time. Additionally, the average baseline value was subtracted for each signal, in order to simulate an optimal scenario where the baseline is approximately zero. This was only done for subject V1, as it is the only case where it was possible to acquire signals from all sensor positions with the textile sensor. This type of analysis was not necessary to be performed for the Ottobock sensor's case, as it is a standard sensor used for prosthetic applications.

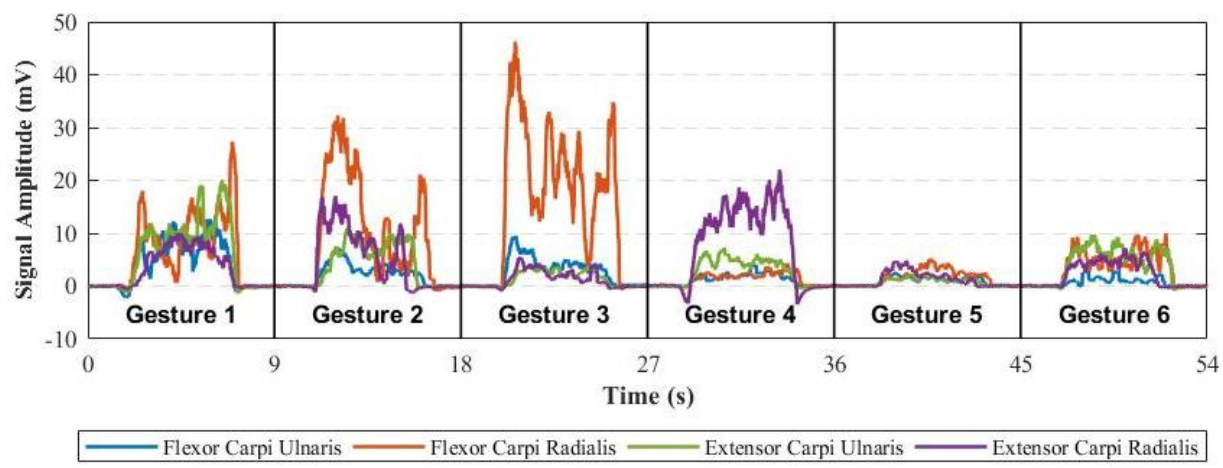

Fig. 12.EMG patterns recorded for each of the six gestures performed by subject V1, taking only into account the first execution of each gesture

\section{Discussion}

Regarding the results presented in Section 3, it is possible to observe in Fig.10 and 11 that the average SNR varied considerably according to the positioning of both sensors, as well as from subject to subject. These differences might have occurred due to several reasons, such as motion artifacts, of which the main sources are the electrode cable and the electrode interface, as the electrodes can move with respect to each other when the subject performs a gesture [17]. There is also the possibility that the sensors were not placed at the optimal muscle position for all subjects, which could have caused different degrees of crosstalk for each subject, a situation that occurs when an undesired EMG signal generated by a nearby muscle group interferes with the signal generated by the desired muscle. Furthermore, the noise in the EMG signal varies according to the subject's characteristics, such as the tissue structure (which includes muscle and fat), blood flow velocity, skin hair, skin temperature, sweat, among others [18]

A priori, a higher average SNR value $(11.74 \pm 8.64 \mathrm{~dB})$ was expected for the signals acquired with the Ottobock sensor, as it is already used for prosthetic applications and optimized in terms of materials and signal conditioning. In fact, as observed in Fig.11, for some sensor positions the average SNR is relatively high, if the results obtained with the textile sensor are taken as comparison. However, for some of the muscle 
positions, the SNR is also considerably low. This variation in the SNR values is well palpable by considering the high standard deviation $(8.64 \mathrm{~dB})$. Besides crosstalk and motion artifacts, the low SNR values for this case could have occurred due to electromagnetic noise, as the human body can behave like an antenna by capturing electric and magnetic radiation from the surrounding environment [18]. It was also possible to observe situations of baseline fluctuation in some of the acquired signals by the Ottobock sensor. This could have happened due the shaking of the electrode cables, as well as electrical drifts in the acquisition equipment [17]. Still regarding the Ottobock sensor, in other situations the signal was relatively weak and there was difficulty in discerning the periods in which the gesture was being performed. Poor skin-electrode interface could have been the issue in these types of occurrences.

Regardless, the total average SNR of the proposed textile and the Ottobock sensor were similar, although a higher variability of values was observed in the latter. These were positive results, considering that the proposed sensor allowed results comparable to the ones obtained with a commercial sensor. However, the issues encountered during the recording with the Ottobock sensor may have favored the textile sensor on the analysis presented throughout the previous section. Despite this, it is important to take into account the situations previously mentioned regarding the impossibility of acquiring any signal with the textile sensor. Nevertheless, the authors consider that a total average of SNR of $10.24 \pm 5.45 \mathrm{~dB}$ shows that the obtained results are promising and leave room for further improvements in future work.

Additionally, the obtaining of visibly different EMG patterns for each gesture, as can be observed in Fig.12, is also a good indicator of the potential of the proposed sensor for classification purposes. For instance, it is interesting to note that the signals corresponding to the sensors placed in the Extensor Carpi Radialis and Extensor Carpi Ulnaris, which are both muscles responsible for wrist extension, present the highest amplitude values for gesture 4, which is a gesture that includes the extension movement of the wrist. An analogous scenario is also observed between the Flexor Carpi Radialis and Flexor Carpi Ulnaris, which are responsible for wrist flexion, and gesture 3. Although this is merely an empirical result, it enables the possibility of using an armband with several textile sensors for controlling a multi-degrees-of-freedom prosthesis, which is the main aim of work being developed by the authors. However, due to the reasons stated in the "Results" Section, it is important to bear in mind that the EMG patterns of each gesture were only plotted for one of the subjects. Thus, for now, this type of results cannot be generalized.

\section{Conclusions and Future Work}

This pilot study proposed to analyze the quality of EMG signals acquired with a textile sensor, in comparison to the quality of the signals acquired with a commercial sensor that is already used in prosthetic control of artificial upper limbs. This analysis was based on the SNR from each recording, which was then averaged per sensor position, for each subject's case, as depicted in Fig.10 and 11. Overall, the textile and the 
Ottobock sensor presented total average SNR values of $10.24 \pm 5.45 \mathrm{~dB}$ and $11.74 \pm 8.64 \mathrm{~dB}$, respectively.

Although the average SNR value obtained with the Ottobock sensor was slightly higher, it was expected that it would be even higher, considering the quality of the sensor. In order to tackle the issues encountered with the use of this sensor, more attention should be given to sensor positioning in order to avoid a high degree of crosstalk, as well as ensuring there is an adequate interface between the sensor and the subject. Electromagnetic noise may also be decreased with adapting filtering strategies [18]. Furthermore, baseline fluctuation can also be corrected with a correct fixation of the electrodes [17].

Nonetheless, in comparison with the Ottobock sensor, the results obtained with the textile sensor were considered to be satisfactory and encouraging for the development of future work. Further improvements on the quality of the signals acquired with the proposed sensor can be achieved by designing strategies to deal with known sources of noise contamination. For instance, the selection of an appropriate electrode size and inter-electrode distance may help decrease the influence of crosstalk $[17,18]$. Also, although the frequency range typically related with motion artifacts $(0-10 \mathrm{~Hz})$ was filtered with the bandpass, it is possible that it could have been one of the sources of noise present in signals. To deal with this, more attention must be given to the design of the electronic circuitry [17]. In order to obtain a detection surface (i.e. electrode-tissue interface area) as good as those present in commercial sensors, the use of a chemical component that can act as an electrolyte has been proposed so that, when combined with the textile electrode, it will be possible to increase the adhesion to the skin.

Another aspect to have into account concerns the impedance of the skin. It is recommended that, for better signal acquisition, impedance should be reduced by removing hair on the skin [19]. However, since a prosthesis is a medical device for daily use, alternatives are needed that minimize impedance without the need to remove them.

It is also important to note that differential amplification and filtering is done on the Ottobock sensor directly, whilst the textile sensor is connected to the signal conditioning circuitry through relatively long wires (about $1 \mathrm{~m}$ ), which is expected to significantly contribute to noise pick-up.

As it was has previously mentioned, five requirements for the execution of the sensor were listed. Regarding adjustability, the choice of an elastic band with touch fasteners allowed the test to be performed on arms with a variation of up to $10 \mathrm{~cm}$, which proves the contemplation of a diverse audience.

In the lightness category, the manufacturer reports that the Ottobock sensor weights $9 \mathrm{~g}$. On the other hand, the textile sensor weighed $17.48 \mathrm{~g}$. For future projects, such variation may be further reduced or even equated if the amount of touch fasteners is succinct, while maintaining the adjustability feature. Even so, the values are considerable significant for their integration into medical devices.

Regarding the last requirement of the sensor design, that is, the replacement of conventional components by textile materials, the test described in this work showed some lack of constancy of signal acquisition by the developed sensor. It is not yet 
known whether this factor is exclusively related to the use of a textile-based electrode. Still, since textiles are flexible materials, they become malleable for application in sockets or insertion in any wearable part, that is, they fulfill the comfort requirement for prolonged use of the medical device, besides being lighter than conventional ones.

In sum, the testing of the proposed prototype presented promising results and they indicate that an armband composed by multiple of these sensors can be a more ergonomic and cheaper alternative to the sensors that are currently used for controlling myoelectric prostheses.

Acknowledgments. This work is financed by Project "Deus ex Machina", NORTE01-0145-FEDER-000026, funded by CCDRN, through Sistema de Apoio à InvestigaçãoCientífica e Tecnológica (ProjetosEstruturados I\&D\&I) of ProgramaOperacional Regional do Norte, from Portugal 2020 and by Project UID/CTM/00264/2019 of 2C2T - Centro de Ciência e TecnologiaTêxtil, funded by National Founds through FCT/MCTES.

\section{References}

1. Jamal, M.Z.: Signal Acquisition Using Surface EMG and Circuit Design Considerations for Robotic Prosthesis. In: Computational intelligence in Electromyography Analysis-A Perspective on Current Applications and Future Challengers. pp. 427-445 (2012).

2. Calado, A., Soares, F., Matos, D.: A Review on Commercially Available Anthropomorphic Myoelectric Prosthetic Hands, Pattern-Recognition-Based Microcontrollers and sEMG Sensors used for Prosthetic Control. IEEE Int. Conf. Auton. Robot Syst. Compet. (ICARSC 2019). 1-6 (2019).

3. Luís, A., Calado, A., Andrade, A.: Comparison between Low-Cost and HighEnd sEMG Sensors for the Control of a Transradial Myoelectric Prosthesis. (2017).

4. Lima, E. de J., Sanca, A., Arabiam, A.: Development of a 3D Printed Prosthetic Myoelectric Hand Driven By Dc Actuators, (2017).

5. Pantelopoulos, A., Bourbakis, N.G.: A survey on wearable sensor-based systems for health monitoring and prognosis. IEEE Trans. Syst. Man Cybern. Part C Appl. Rev. 40, 1-12 (2010).

6. Stoppa, M., Chiolerio, A.: Wearable electronics and smart textiles: A critical review. Sensors (Switzerland). 14, 11957-11992 (2014).

7. Zhang, H., Tian, L., Zhang, L., Li, G.: Using textile electrode EMG for prosthetic movement identification in transradial amputees. 2013 IEEE Int. Conf. Body Sens. Networks, BSN, (2013).

8. Li, G., Geng, Y., Tao, D., Zhou, P.: Performance of electromyography recorded using textile electrodes in classifying arm movements. In: Proceedings of the Annual International Conference of the IEEE Engineering in Medicine and Biology Society, EMBS. pp. 4243-4246. IEEE EMBS, 
Boston (2011).

9. Lee, S., Kim, M.O., Kang, T., Park, J., Choi, Y.: Knit Band Sensor for Myoelectric Control of Surface EMG-Based Prosthetic Hand. IEEE Sens. J. 18, 8578-8586 (2018).

10. Biddiss, E., Chau, T.: Upper limb prosthesis use and abandonment: A survey of the last 25 years. Prosthet. Orthot. Int. 31, 236-257 (2007).

11. Cordella, F., Ciancio, A.L., Sacchetti, R., Davalli, A., Cutti, A.G., Guglielmelli, E., Zollo, L.: Literature review on needs of upper limb prosthesis users, (2016).

12. Biomedical Health and Research Program (BIOMED II) of the European Union.: SENIAM, http://www.seniam.org/.

13. Paiva, A., Ferreira, F., Catarino, A., Carvalho, M., Carvalho, H.: Design and characterization of a textile extension sensor for sports and health applications. IOP Conf. Ser. Mater. Sci. Eng. 459, 0-6 (2018).

14. Delagi, E.F., Iazzetti, J., Perotto, A.O., Morrison, D.: Anatomical guide for the Electromyographer: the limbs and trunk. Charles C Thomas Publisher LTD., New York (2011).

15. Kamen, G., A. Gabriel, D.: Essentials of Electromyography. Human Kinects (2009).

16. Konrad, P.: The ABC of EMG. A Pract. Introd. to Kinesiol. ... 1-60 (2005).

17. H, A. V: A Review on Noises in EMG Signal and its Removal. Int. J. Sci. Res. Publ. 7, 23 (2017).

18. Chowdhury, R.H., Reaz, M.B.I., Bin Mohd Ali, M.A., Bakar, A.A.A., Chellappan, K., Chang, T.G.: Surface electromyography signal processing and classification techniques. Sensors (Switzerland). 13, 12431-12466 (2013).

19. Winter, D.A.: Biomechanics and Motor Control of Human Movement. John Wiley \& Sons (2009). 